\title{
Ultrafast Thermo-Optical Dynamics of a Single Metal Nano-Object
}

\author{
Romain Rouxel ${ }^{1}$, Michele Diego ${ }^{1}$, Fabio Medeghini ${ }^{1+}$, Paolo Maioli ${ }^{1}$, Francesco Rossella ${ }^{2}$, Fabrice \\ Vallée ${ }^{1}$, Francesco Banfi ${ }^{1}$, Aurélien Crut $^{{ }^{*}}$ and Natalia Del Fatti ${ }^{1}$ \\ ${ }^{1}$ FemtoNanoOptics group, Université de Lyon, CNRS, Université Claude Bernard Lyon 1, Institut Lumière \\ Matière, F-69622 Villeurbanne, France \\ ${ }^{2}$ NEST, Scuola Normale Superiore and Istituto Nanoscienze-CNR, Piazza S. Silvestro 12, I-56124 Pisa, \\ Italy
}

\section{ABSTRACT}

Single-particle optical spectroscopy methods have enabled quantitative investigations of the optical, electronic and vibrational responses of nano-objects in the recent years. In this work, single-particle pump-probe optical spectroscopy was exploited to investigate the cooling dynamics of individual gold nanodisks supported on a sapphire substrate. The measured time-resolved signals are shown to directly reflect the temporal evolution of the nanodisk temperature following its sudden excitation. The single-particle character of the experiments enables a quantitative analysis of the amplitudes of the measured time-resolved signals, allowing to rationalize their large probe wavelength-dependence. The measured cooling kinetics mainly depends on nanodisk thickness and to a much lesser extent on diameter, in agreement with numerical simulations based on Fourier law of heat diffusion, also accounting for the presence of a thermal resistance at the interface between the nanodisks and their substrate. For the explored diameter range $(60-190 \mathrm{~nm})$, the nanodisk cooling rate is limited by heat transfer at the gold-sapphire interface, whose thermal conductance can be estimated for each investigated nanodisk.

KEYWORDS: nanoscale heat transfer, single-particle spectroscopy, metal nano-objects, Kapitza resistance, thermoplasmonics 


\section{INTRODUCTION}

A detailed understanding of nanoscale heat transfer is required in many technologies. It is for instance essential to manage the crucial problem of heat dissipation from nanoscale components in nanoelectronics, to create nanostructured materials with good thermoelectric properties and to maximize the efficiency of nanoparticle-based photothermal therapies. ${ }^{1-4}$ Nanoscale heat transfer fundamentally differs from that at macroscopic scale for two major reasons. First, the thermal resistances limiting heat transfer at interfaces ("Kapitza resistances"5,6) play a much greater role in the former case, and rule the cooling rate of small nanostructures. ${ }^{7-10}$ Second, Fourier law of heat conduction, which assumes a diffusive motion of heat carriers, ceases to be applicable to describe heat propagation over distances smaller than their characteristic mean free paths (ballistic regime) ) $^{11,12}$ and in situations where the causality relation between the temperature gradient and heat flux becomes manifest (hydrodynamic regime). ${ }^{13,14}$

A lot of the current understanding of nanoscale heat transfer has been obtained using noncontact optical pump-probe techniques. The main idea of this approach consists in investigating the dissipation of the energy injected by a pump light beam in a sample by monitoring the induced modifications of the sample optical properties, e.g. by measuring the transmission or reflection of a probe beam. This general strategy can be implemented in various ways, differing by the types of light sources, spectroscopy methods and samples used. For instance, pump-probe techniques can work either in the frequency domain ${ }^{15,16}$ (modulating pump power, the use of continuous beams being possible in this case) or time domain ${ }^{17}$ (using time-delayed pump and probe pulses, which can be spatially overlapped or separated), and can be applied to both bulk and nanostructured materials, provided that they are partly absorbing. ${ }^{18,19}$

Time-resolved experiments involving absorbing nanostructures (playing the role of both heaters and thermometers) dispersed in a dielectric environment are particularly interesting to study nanoscale heat transfer. Indeed, the sizes of the heated volumes correspond in this case to those of the absorbing nano-objects (which can be of the order of a few or a few tens of $\mathrm{nm}$ ), rather than that of the pump beam, whose lateral size is limited by diffraction to about half of its wavelength, i.e. a few hundreds of $\mathrm{nm}$ (and which constitutes the relevant length scale for heating of bulk materials and thin films). This approach has for instance been used to measure the thermal conductances at the interface between metal nanoparticles and their environment, and to investigate the dependence of these conductances on composition of nanoparticles, environment and interfaces (e.g., nature and length of surfactant molecules). ${ }^{7,20-23}$ The relation between the measured time-resolved optical signals and the thermal kinetics in the nanoparticles and their local environment has also been recently modeled, ${ }^{21,24,25}$ 
allowing more precise estimations of interface conductances in cases where environment heating significantly influences time-resolved signals. Additionally, some time-resolved experiments performed on arrays of metal nanowires patterned on crystalline substrates with high phonon mean free paths (e.g. sapphire and silicon) have demonstrated the failure of Fourier law at the nanoscale. ${ }^{12,26-29}$

All the studies mentioned above were performed on ensembles of nanostructures. The signals measured in these investigations thus represent an average over nanostructures affected by an unavoidable dispersion of their morphology and thermal contact with the environment. This approach makes analysis of the amplitude of time-resolved signals extremely challenging, as it would require to precisely know the density of the nanoparticles and their size dispersion, and to take into account the different efficiencies with which their heating is generated and probed (due to their different locations in the non-uniform pump and probe beams, and to the dispersion of their optical responses resulting from their morphological differences). Single-particle methods, which have already been applied to study the linear optical response,,$^{30,31}$ internal thermalization ${ }^{32}$ and acoustic vibrations $s^{33-36}$ of metal nano-objects, constitute an ideal way to overcome these limitations. In this work, we apply singleparticle time-resolved spectroscopy to the study of the cooling dynamics of individual metal nanoparticles: gold nanodisks (NDs) supported on sapphire. We report quantitative investigations of the sensitivity of the measured signals to ND heating, by measuring the proportionality factor between optical cross-section changes and nanoparticle temperature rise. This sensitivity is shown to strongly depend on the probe wavelength used, in agreement with the results of previous thermo-optical models. ${ }^{21,24}$ By performing experiments on a large number of individual NDs with different diameters and thicknesses, we also characterize the size-dependence of ND cooling dynamics. Finite-element modeling (FEM) was used to demonstrate that ND cooling dynamics are essentially determined by the thermal resistance at the gold-sapphire interface, whose value can be determined by comparison between the measured and simulated signals.

\section{EXPERIMENTAL AND THEORETICAL METHODS}

Nanodisk fabrication and morphological characterization. Gold NDs with a diameter spanning the $D=60-190 \mathrm{~nm}$ range were nanopatterned by a combination of electron beam lithography, $\mathrm{Au}$ thermal evaporation and lift-off techniques on the optically polished surface of a $480 \mu \mathrm{m}$ thick (0001) $\alpha-\mathrm{Al}_{2} \mathrm{O}_{3}$ single crystal sapphire substrate. ${ }^{36,37} \mathrm{~A}$ large $(10 \mu \mathrm{m})$ separation between adjacent NDs was chosen so as to allow optical investigation of a single ND and to avoid optical and thermal inter-ND 
coupling effects. Atomic force and scanning electron microscopy characterizations of the NDs were performed to estimate the thicknesses and diameters of the produced NDs, respectively.

Spatial modulation microscopy/spectroscopy. Localization and linear optical characterization of individual NDs were performed by spatial modulation spectroscopy (SMS). ${ }^{38}$ This microscopy/spectroscopy far-field optical technique is based on the periodic displacement of a single nano-object in the focal spot of a tightly focused light beam, which induces a modulation of the transmitted light power (Fig. 1a). The measured signal being related to the extinction cross-section $\sigma_{\text {ext }}$ of the investigated nano-object via accessible experimental parameters (size of the illuminating spot and amplitude of the spatial modulation), SMS enables $\sigma_{\text {ext }}$ to be quantitatively determined as a function of the illumination wavelength $\lambda$ and light polarization angle $\theta$. SMS experiments were performed using a tunable Ti:sapphire oscillator combined with a visible optical parametric oscillator and a frequency-doubling BBO crystal as a light source, allowing optical studies in the $375-1040 \mathrm{~nm}$ wavelength range. The light beam delivered by this source was focused on the sample by a $100 \mathrm{X}$ microscope objective, the direction of its linear polarization being controlled by a wire grid polarizer. The size of the laser spot at the sample surface, close to the diffraction limit (about $0.7 \lambda$ full-width at half-maximum), was precisely determined by two-dimensional fitting of the SMS images of small NDs obtained using a translation stage. Spatial modulation of the sample was performed at $f=1.5 \mathrm{kHz}$ frequency with $300 \mathrm{~nm}$ modulation amplitude and lock-in detection at 2f. Quasi-circular NDs were identified from the weak dependence of their extinction on $\theta .{ }^{37}$

Time-resolved experiments. The ultrafast dynamics of individual NDs were monitored using single-particle femtosecond pump-probe spectroscopy, ${ }^{32,39}$ an approach based on the illumination of the investigated nanoparticle with two spatially overlapping, tightly focused and time-delayed femtosecond light pulses, referred to as pump and probe (Fig. 1C). Time-resolved measurements on individual NDs were performed by combining the SMS microscope with a two-color pump-probe setup based on the femtosecond laser source described in the previous paragraph, delivering 150 fs pulses. The oscillator pulse train was split in two parts to generate the pump and probe beams. The pump beam was produced using second harmonic generation (yielding $\lambda_{\mathrm{pp}}$ in the $375-510 \mathrm{~nm}$ range), while the probe beam was either the signal from the Ti:sapphire oscillator (with $\lambda_{\mathrm{pr}}=690-1040 \mathrm{~nm}$ in this case) or the output of the optical parametric oscillator $\left(\lambda_{\text {pr }}=500-730 \mathrm{~nm}\right)$. A mechanical chopper operating at $30 \mathrm{kHz}$ was used to modulate the pump power. The pump-induced relative changes of probe beam transmission, $\Delta \operatorname{Tr} / \mathrm{Tr}$, were measured using synchronous detection as a function of the time interval separating pump and probe pulses, controlled by a mechanical delay line. This raw measured signal is directly related to the transient changes of the extinction cross-section $\sigma_{\text {ext }}\left(\lambda_{\text {pr }}\right)$ of the investigated nano-object at probe wavelength $\lambda_{\text {pr }}$ via $\Delta \operatorname{Tr} / T r=-\Delta \sigma_{\text {ext }}\left(\lambda_{\text {pr }}\right) / S_{p r}$, with $S_{p r}=\pi d_{F W H M}{ }^{2} /(4$ 
$\ln 2$ ) the area of the probe spot and $d_{\mathrm{FWHM}}$ its full width at half maximum. ${ }^{32}$ As $\mathrm{S}_{\mathrm{pr}}$ is an experimentally accessible parameter, these experiments provide access to the ultrafast variations of $\Delta \sigma_{\text {ext }}$ as a function of the pump-probe delay. Frequent optical realignments were performed so as to maintain the pump and probe beams centered on the investigated ND.

Optical simulations. Modeling of the ND plasmonic response was performed by FEM, using the RF module of the COMSOL commercial software. Gold NDs were described as circular cylinders characterized by their height $h$ and diameter $D$. Their inhomogeneous environment resulting from the fact that they are supported on a sapphire substrate was explicitly included in the model, refractive indexes of 1.76 and 1 being used for the sapphire substrate and the above medium, respectively. $A$ perfectly matched layer was used around the simulation domain to avoid spurious finite-size effects. Gold dielectric function $\varepsilon$ was modeled based on Johnson and Christy tables of bulk gold dielectric function $\varepsilon^{\mathrm{JC} 40}$ (the most common choice in the literature although other tables are available ${ }^{41,42}$ ). $\varepsilon$ was written as $\varepsilon(\omega)=\varepsilon^{\lrcorner \mathcal{C}}(\omega)+\omega_{p}{ }^{2} /\left(\omega\left(\omega+i \gamma_{0}\right)\right)-\omega_{p}{ }^{2} /(\omega(\omega+i \gamma))$, which corresponds to describing the dielectric response of gold conduction electrons by a Drude term ${ }^{30}$ involving the bulk gold plasma frequency $\omega_{p}$ $\left(\hbar \omega_{P}=9.01 \mathrm{eV}\right)$ and an optical scattering rate $\gamma$ of the conduction electrons larger than that of bulk gold $\left(\hbar \gamma_{0} \approx 50 \mathrm{meV}\right) \cdot{ }^{37} \mathrm{ND}$ illumination by a linearly polarized plane wave was considered. Absorption and scattering cross-sections were deduced from the computed electric field and summed to yield the extinction one. Simulations were performed for different $D$ and $h$ values until a good agreement with the investigated experimental spectrum was reached, as shown in Fig. $1 \mathrm{a}$. The $\partial \sigma_{\mathrm{ext}} / \partial \varepsilon_{1}$ and $\partial \sigma_{\mathrm{ext}} / \partial \varepsilon_{2}$ partial derivatives (Fig. 1b) were deduced from the calculations leading to an optimal reproduction of experimental spectra by first reproducing them with slightly modified $\varepsilon_{1}$ or $\varepsilon_{2}$ values and then performing discrete derivation.

Cooling dynamics simulations. The cooling dynamics of gold NDs were modeled using the Heat Transfer module of COMSOL. Gold NDs were described as perfect cylinders. The sapphire substrate was explicitly included in the simulations as a half-sphere of $1 \mu \mathrm{m}$ radius (large enough to ensure that the choice of boundary conditions at its external border does not influence the computed dynamics). Gold and sapphire were described using their bulk thermal parameters, i.e. $\mathrm{c}_{\mathrm{Au}}=2.5 \mathrm{~J} \cdot \mathrm{cm}^{-3} \cdot \mathrm{K}^{-1}$ and $\mathrm{C}_{\mathrm{A} 1203}=3.1 \mathrm{~J} \cdot \mathrm{cm}^{-3} \cdot \mathrm{K}^{-1}$ volumetric heat capacities, and $\Lambda_{\mathrm{Au}}=317 \mathrm{~W} \cdot \mathrm{m}^{-1} \cdot \mathrm{K}^{-1}$ and $\Lambda_{\mathrm{A} 1203}=25 \mathrm{~W} \cdot \mathrm{m}^{-1} \cdot \mathrm{K}^{-1}$ thermal conductivities (note that the small temperature excursions in our experiments make unnecessary to consider their temperature-dependence). Perfect contact and finite thermal conductance G were assumed at their interface. Simulations were performed in the time domain with a $10 \mathrm{ps}$ time step, the axial symmetry of the system being exploited to reduce computational costs. $\mathrm{A} \Delta \mathrm{T}_{0}$ initial temperature 
rise was defined in the ND. ND temperature was seen to remain quasi-uniform throughout the cooling process, as expected from a $B i=h G / \Lambda_{A u}<<1$ Biot number.

\section{RESULTS AND DISCUSSION}

Two samples of sapphire-supported Gold NDs with different ND thicknesses ( $h \approx 18$ and $40 \mathrm{~nm}$, with ND diameters ranging from 60 to $190 \mathrm{~nm}$ in both cases) were made. The extinction spectra of the investigated single NDs were determined using SMS (Fig. 1a). ${ }^{30,38}$ As discussed in detail in a recent paper, ${ }^{37}$ combination of SMS measurements with numerical modeling of ND optical response constitutes a powerful tool for the morphological characterization of single NDs, especially since their electron microscopy imaging is challenging (large substrate thickness preventing the use of transmission electron microscopy and substrate charging degrading the resolution of scanning electron microscopy images). On the one hand, polarization-dependent extinction measurements allow the detection of minute deviations of actual ND shapes from the targeted cylindrical ones, as they induce a polarization-dependent extinction cross-sections and lift the degeneracy of ND in-plane dipolar localized surface plasmon resonances (SPRs). ${ }^{37}$ On the other hand, comparison of the measured extinction spectra with those generated using optical numerical simulations allows one to estimate the dimensions of the investigated NDs, which may differ from those targeted in the nanofabrication process. ${ }^{37}$ The extinction spectrum of one of the investigated NDs (referred to as ND1 in the following) is shown in Fig. 1a as an example. It displays a marked resonance near $700 \mathrm{~nm}$ wavelength, associated to the in-plane SPR of ND1. This spectrum was found to be quasi-independent of polarization, thus suggesting a quasi-circular ND section. ND1 extinction spectrum could be well reproduced by FEM simulations accounting for the sapphire substrate supporting the NDs, using $D=101 \mathrm{~nm}$ and $\mathrm{h}=21 \mathrm{~nm}$ for the ND diameter and thickness (note that using an optical scattering rate $\gamma$ of conduction electrons larger than that of bulk gold $\gamma_{0}$, with $\hbar \gamma_{0} \approx 50 \mathrm{meV}$, is necessary to reproduce the measured SPR width, with $\hbar \gamma=\hbar \gamma_{0}+100 \mathrm{meV}$ for the ND shown in Fig. 1a, as in previous analyses on NDs of similar sizes ${ }^{37}$; the large SPR width probably results from the high polycrystallinity of nanostructures produced using electron beam lithography). These deduced $D$ and $h$ values, are in reasonable agreement (about $10 \%$ larger) with those measured with atomic force and scanning electron microscopies, respectively. FEM simulations also enable to determine the sensitivity of the extinction spectra of NDs to changes in the gold dielectric function $\varepsilon=\varepsilon_{1}+i \varepsilon_{2}$, which is an essential ingredient to analyze the effect of ND heating on its optical response, as discussed in detail below. This sensitivity is quantified by $\partial \sigma_{\text {ext }} / \partial \varepsilon_{1}$ and $\partial \sigma_{\text {ext }} / \partial \varepsilon_{2}$ partial derivatives (Fig. 1b). These derivatives both present enhanced amplitudes in the SPR 
domain but display different spectral shapes, reflecting the fact that $\varepsilon_{1}$ and $\varepsilon_{2}$ mostly affect SPR position and width, respectively.

The ultrafast dynamics of individual NDs were monitored using single-particle femtosecond pump-probe spectroscopy, ${ }^{32,39}$ an approach based on the illumination of the investigated nanoparticle with two spatially overlapping, tightly focused and time-delayed femtosecond light pulses, referred to as pump and probe (Fig. 1C, also see the Methods part). The transient $\Delta \sigma_{\text {ext }}$ variations measured using $\lambda_{\mathrm{pp}}=410 \mathrm{~nm}$ and $\lambda_{\mathrm{pr}}=690 \mathrm{~nm}$ pump and probe wavelengths for ND1 are presented as an example in Fig. 1c. The measured signal contains signatures of the sample excitation by pump pulse absorption and the induced relaxation mechanisms (processes 1-3 in Fig. 1c). ${ }^{39}$ The sharp peak observed at short timescales reflects the gold ND ultrafast excitation and internal thermalization on $\mathrm{a} \approx 1 \mathrm{ps}$ timescale by electron-electron and electron-phonon scattering mechanisms (process 1 in Fig. 1c). This impulsively launches, by a displacive excitation mechanism induced by thermal dilation, ND acoustic vibrations, which appear on nanosecond timescales as damped oscillations in time-resolved signals (process 2 in Fig. 1c) and have been the scope of recent studies. ${ }^{36,37}$ This feature partly overlaps with a monotonic decay of the signal associated to ND cooling (i.e., dissipation of the thermal energy injected by the pump pulse, also occurring on a nanosecond timescale, process 3 in Fig. 1c). The contribution of the cooling process to the time-resolved signals can be obtained upon subtraction of ND internal thermalization and vibrational response contributions, respectively fitted by an exponential decay and a sum of damped sinusoids, each corresponding to a detected vibrational mode. The deduced thermal component, shown in the inset of Fig. 1c, presents a quasi mono-exponential decay over the whole investigated range of pump-probe delays (up to $2.5 \mathrm{~ns}$ ). More generally, mono-exponential decays were observed at long time scales (>500 ps) for all signals, but deviations from a mono-exponential behavior were observed at short time scales for some of the investigated NDs (in particular in the case of excitation with high pump fluence, as discussed below). All thermal components could however be accurately reproduced using a biexponential decay function $A_{1} \exp \left(-t / \tau_{1}\right)+A_{2} \exp \left(-t / \tau_{2}\right)$, with $A_{1}>A_{2}$ amplitudes and characteristic times of the order of $\tau_{1} \approx 2 \mathrm{~ns}$ and $\tau_{2} \approx 200 \mathrm{ps}$ in the case of $\mathrm{h}=18 \mathrm{~nm}$ NDs.

Time-resolved signals being averaged over multiple acquisitions, care must be exerted to ensure that no modification of the ND thermal dynamics occurs during the course of the experiments. Controlling the pump-induced initial ND heating is critical in this context, as shown in Fig. 2 for experiments on ND2, a ND fabricated with the same parameters as ND1. For a pump beam fluence at the particle position of $F_{p p}=1 \mathrm{~J}^{-2}$ (deduced from measurements of pump beam power and spot size), successive acquisitions were reproducible, corresponding to a quasi-exponential response with $A_{1} / A_{2}=4.4$ (black line in Fig. 2a). However, increasing pump fluence to $F_{p p}=2.7 \mathrm{~J} . \mathrm{m}^{-2}$ led to marked 
modifications of the cooling dynamics (red line in Fig. 2a), manifesting in biexponential fits with $A_{1} / A_{2}$ ratio reduced to 1.8. These changes were preserved after return to the initial lower pump fluence (blue line in Fig. 2a). Additionally, they were correlated with a permanent modification of the extinction spectrum of the ND (Fig. 2b), whose SPR became red shifted and with larger maximal amplitude after ND2 exposure to the $2.7 \mathrm{~J}^{-2} \mathrm{~m}^{-2}$ fluence. In the following, thermal investigations were performed in a low excitation regime to avoid such perturbations.

The initial temperature rise of an internally thermalized metal nanoparticle corresponding to a given $\mathrm{F}_{\mathrm{pp}}$ value was estimated as $\Delta \mathrm{T}_{0}=\mathrm{u}_{\mathrm{abs}} / \mathrm{C}_{\mathrm{Au}}$, with $\mathrm{C}_{\mathrm{Au}}$ the volumetric heat capacity of gold $\left(\mathrm{C}_{\mathrm{Au}}=2.5\right.$ $\left.J . \mathrm{cm}^{-3} \cdot \mathrm{K}^{-1}\right)$ and $\mathrm{u}_{\mathrm{abs}}=\sigma_{\mathrm{abs}}\left(\lambda_{\mathrm{pp}}\right) \mathrm{F}_{\mathrm{pp}} / \mathrm{V}$ the absorbed energy density, determined by pump fluence $\mathrm{F}_{\mathrm{pp}}, \mathrm{ND}$ volume $V$ and absorption cross-section at pump wavelength $\sigma_{a b s}\left(\lambda_{p p}\right) . V$ and $\sigma_{a b s}\left(\lambda_{p p}\right)$ are obtained from optical FEM calculations. Specifically, $\mathrm{V}$ is computed using the sizes used in the simulation to achieve an optimal reproduction of the experimental extinction spectrum (Fig. 1a). FEM simulations also allow to separate the contributions of absorption and scattering to extinction, and thus to estimate $\sigma_{\text {abs }}\left(\lambda_{\text {pp }}\right)$. This analysis led to $\Delta T_{0} \approx 15$ and $40 \mathrm{~K}$ for the two fluence values used in Fig. 2. For the highest fluence, the ultrafast initial electronic excitation and subsequent electron-lattice thermalization at $T_{0}+\Delta T_{0}$ appear sufficient to induce permanent modifications in the ND or its local environment. Understanding the precise mechanisms underlying this effect would require further investigations, but is out of the scope of this paper, where only $\Delta T_{0}$ values below $15 \mathrm{~K}$ were considered after these preliminary studies, so as to avoid modification of ND linear and ultrafast optical responses during the course of the experiments.

The effect of probe wavelength on the time-resolved signals measured on ND1 is illustrated in Fig. 3. In our experimental setup based on a Ti:sapphire oscillator, either frequency-doubled or driving an optical parametric oscillator (see the Methods part), the wavelengths of the pump and probe beams cannot be chosen fully independently; therefore their parameters could not be kept constant throughout this series of experiments. In order to isolate the effect of probe wavelength from that of pump excitation, the measured transient extinction cross-section changes $\Delta \sigma_{\text {ext }}$ were divided by the estimated initial temperature rise $\Delta \mathrm{T}_{0}$, with $\Delta \mathrm{T}_{0} \approx 10 \mathrm{~K}$ for the $\mathrm{F}_{\mathrm{pp}} \approx 0.7 \mathrm{~J} / \mathrm{m}^{2}$ typical pump beam fluence used in these experiments. Fig. 3a shows that the probe wavelength considerably affects the amplitude of time-resolved signals, but weakly their temporal dynamics. This is best appreciated by separately comparing the temporal dynamics (inset of Fig. 3a) and amplitudes (Fig. 3a-b) of the thermal components of the measured time-resolved signals, obtained by subtraction of the internal thermalization and vibrational contributions. Indeed, the inset of Fig. 3a shows that all thermal components overlap once normalized by their initial value, while Fig. 3b allows one to appreciate their variations of sign and amplitude in the spectral range of the ND SPR (Fig. 1a). 
These observations can be understood considering the simplified situation of uniform temperatures in both the nanoparticle and its local surrounding medium (assumed homogeneous here), noted $T_{p}$ and $T_{m}{ }^{21,24}$ (environment heterogeneity and $T_{m}$ spatial variations being conversely included in the more complete FEM simulations of ND thermal dynamics presented below). In this simplified case, time- and probe wavelength-dependent transient extinction changes are related, to first order, to the temporal evolutions of $T_{p}$ and $T_{m}$ via

$$
\Delta \sigma_{e x t}\left(\lambda_{p r}, t\right)=A_{p}\left(\lambda_{p r}\right) \Delta T_{p}(t)+A_{m}\left(\lambda_{p r}\right) \Delta T_{m}(t)
$$

with

$$
\begin{aligned}
& A_{p}(\lambda)=\frac{\partial \sigma_{e x t}}{\partial \varepsilon_{1}}(\lambda) \frac{d \varepsilon_{1}}{d T_{p}}(\lambda)+\frac{\partial \sigma_{e x t}}{\partial \varepsilon_{2}}(\lambda) \frac{d \varepsilon_{2}}{d T_{p}}(\lambda) \\
& A_{m}(\lambda)=\frac{\partial \sigma_{e x t}}{\partial \varepsilon_{m}}(\lambda) \frac{d \varepsilon_{m}}{d T_{m}}(\lambda)
\end{aligned}
$$

where $\varepsilon=\varepsilon_{1}+i \varepsilon_{2}$ is the complex dielectric function of the metal nanoparticle, and $\varepsilon_{m}$ that of its environment, assumed real. Eq. 1 shows that transient extinction changes generally reflect a combination of the thermal dynamics in the nanoparticle and its environment, with $\lambda_{\text {pr-dependent }}$ weights. This may lead to a complex dependence on probe wavelength of time-resolved signals, as was experimentally demonstrated in the case of gold nanoparticles in ethanol. ${ }^{21}$ This effect originates from the different dynamics of the temperatures in the nanoparticle and its local environment in the context of actual time-resolved experiments. Indeed, the former is suddenly increased by pump pulse absorption and monotonously decays towards its equilibrium value, while the latter reaches its maximum with a position-dependent delay, due to the time needed for the heat generated in the nanoparticle to be transferred and to propagate into its environment. ${ }^{21,24}$

In the present study, the similarities of the dynamics measured using a large number of probe wavelengths and the monotonous decay of $\left|\Delta \sigma_{\text {ext }}\right|$ as a function of time demonstrated by the inset of Fig. 3a imply that sapphire substrate heating has a negligible influence on the measured signals. This can be ascribed to the weak sensitivity of sapphire dielectric function to temperature changes, with $\mathrm{d} \varepsilon_{\mathrm{m}} / \mathrm{dT} \approx 4.10^{-5} \mathrm{~K}^{-1}$ in the visible range, ${ }^{43}$ to be compared to the $10^{-3} \mathrm{~K}^{-1}$ typical values of $\left|\mathrm{d} \varepsilon_{1} / \mathrm{dT}\right|$ and $\left|\mathrm{d} \varepsilon_{2} / \mathrm{dT}\right|$ for gold in the same spectral range. ${ }^{44,45}$ Additionally, the high thermal conductivity of sapphire $\left(25 \mathrm{~W} . \mathrm{m}^{-1} . \mathrm{K}^{-1}\right)$ favors heat dissipation in the substrate away from the ND vicinity, and thus limits the temperature rise in this region.

The negligible contribution of environment heating to time-resolved signals means that the heating-induced changes of the ND extinction cross-section are proportional to the ND temperature rise at all times (this temperature being well-defined for pump-probe delays exceeding 
the few ps duration required for complete ND thermalization by electron-electron and electronphonon interaction processes). In particular, the $A_{p}$ coefficient entering eq. 1 can be computed as the ratio between the initial amplitude of the thermal signal and the estimated initial ND temperature rise $\Delta T_{0}$. The $A_{p}$ spectrum of ND1 retrieved from time-resolved experimental signals is shown in Fig. 3b. Its spectral shape resembles that of the $\partial \sigma_{\mathrm{ext}} / \partial \varepsilon_{2}$ derivative shown in Fig. $1 \mathrm{~b}$ : it displays large and negative values near the central position of the SPR (dashed blue line in Fig. $3 b-c$ ) and smaller, positive values on the SPR sides.

$A_{p}$ can also be theoretically estimated from eq. 2 , which requires knowledge of $\partial \sigma_{\text {ext }} / \partial \varepsilon_{1,2}$ and $\mathrm{d} \varepsilon_{1,2} / \mathrm{dT}$ derivatives for the investigated gold ND. The determination of $\partial \sigma_{\mathrm{ext}} / \partial \varepsilon_{1,2}$ (Fig. 1b) from optical FEM computations has been presented above. The temperature dependence of gold dielectric function has been the object of multiple recent measurements and models. ${ }^{44-50}$ The thermal derivatives $d \varepsilon_{1,2} / d T$ extracted from these measurements strongly depend on the type of gold sample used (continuous or granular, annealed or not, deposited thickness) and on temperature. As a result, the values of the $d \varepsilon_{1,2} / d T$ coefficients of gold reported in the literature are dispersed, in particular for the $d \varepsilon_{1} / d T$ derivative, as $\varepsilon_{1}$ presents large values but weak relative changes with temperature in the visible range. To evaluate the impact of these uncertainties on $A_{p}$ spectra, two different $d \varepsilon_{1,2} / d T$ tables were used for computing $A_{p}$ (Fig. 3c): one experimentally determined by Wilson and Cahill, ${ }^{44}$ and one theoretically estimated from the expected temperature dependences of Drude and interband components of gold dielectric function, as discussed in ref. ${ }^{45}$ (see figure 6 of this reference). The $d \varepsilon_{1,2} / d T$ values present significant differences in the red/near infrared range relevant to the present experiments. For instance, at $800 \mathrm{~nm}$ wavelength, the former table yield $\mathrm{d} \varepsilon_{1} / \mathrm{dT} \approx 3.10^{-5} \mathrm{~K}^{-1}$ and $\mathrm{d} \varepsilon_{2} / \mathrm{dT} \approx 2.10^{-3} \mathrm{~K}^{-1}$, ${ }^{44}$ while the latter one corresponds to $\mathrm{d} \varepsilon_{1} / \mathrm{dT} \approx 1.10^{-3} \mathrm{~K}^{-1}$ and $\mathrm{d} \varepsilon_{2} / \mathrm{dT} \approx 3.10^{-3} \mathrm{~K}^{-1} .{ }^{45} \mathrm{As}$ a result, the computed $\mathrm{A}_{\mathrm{p}}$ spectra differ depending on the chosen table (Fig. 3c). However, they both present spectral variations in good agreement with the experimental results, close to those of the $\partial \sigma_{\text {ext }} / \partial \varepsilon_{2}$ derivative (Fig. $1 \mathrm{~b}$ ) which result from the fact that $\left|\mathrm{d} \varepsilon_{2} / \mathrm{dT}\right|>\left|\mathrm{d} \varepsilon_{1} / \mathrm{dT}\right|$ in the investigated spectral range for both considered $d \varepsilon_{1,2} / d T$ tables (see eq. 2). The measured and computed $A_{p}$ amplitudes, which have maximal values of a few tens of $\mathrm{nm}^{2} \cdot \mathrm{K}^{-1}$ also show a good quantitative agreement considering the high number of parameters involved in the analysis and the large uncertainties affecting $\mathrm{d} \varepsilon_{1,2} / \mathrm{dT}$ coefficients.

The proportionality between the measured time-resolved signals and ND transient temperature rise was exploited for systematic investigations of the size-dependence of ND cooling dynamics. Time-resolved signals were collected on 60 different NDs of different sizes. In all the experiments, sample illumination with a $\lambda_{\mathrm{pp}}=410 \mathrm{~nm}$, low fluence $\left(<1 \mathrm{~J} . \mathrm{m}^{-2}\right)$ pump beam was used in order to achieve moderate $(\approx 10 \mathrm{~K})$ initial temperature rises and avoid ND modification throughout the 
measurements (Fig. 2). Since probe wavelength choice is not critical to achieve $\Delta \sigma_{\text {ext }} \propto \Delta T_{p}$ (as shown by Fig. $3 \mathrm{~b}$ ), a $\lambda_{\mathrm{pr}}=510 \mathrm{~nm}$ wavelength away from the ND SPR domain (Fig. 1a) was used as it reduces the relative contribution of ND acoustic vibrations to the measured time-resolved signals. All bare time-resolved signals were processed by subtraction of internal thermalization and vibrational signatures, as in Figs. 1a and 3a.

Fig. 4a presents representative normalized thermal signals obtained for NDs with different thickness ( $h=18$ and $h=40 \mathrm{~nm}$, average thicknesses of the two samples used determined by AFM) and diameter (from 60 to $180 \mathrm{~nm}$ ), showing that their cooling kinetics is strongly dependent on $\mathrm{h}$ and weakly dependent on $D$ in this size range. All measured thermal components present an exponential decay at large time scales ( $>500$ ps delays), with an associated decay time $\tau_{1}$ of about 0.7 and $1.7 \mathrm{~ns}$ for $h=18$ and $h=40 \mathrm{~nm}$ NDs, respectively (as discussed above, some NDs present a biexponential dynamics, whose description requires a second characteristic time $\tau_{2}<<\tau_{1}$ ). Such exponential behaviour suggests an interface-limited cooling dynamics. ${ }^{7,8}$ Indeed, in the case of a Biot number $B i=h G / \Lambda_{A u}<<1$ (with $\Lambda_{\mathrm{Au}}$ the gold ND thermal conductivity), which guarantees that the ND remains isothermal throughout the thermal relaxation process, and of a substrate heat conductivity high enough to ensure negligible modification of substrate temperature near the ND being cooled (two relevant assumptions in the case of $A u$ NDs on sapphire), integrating the interfacial heat current density $J_{\text {int }}=G \Delta T_{p}$ (Fig. $4 b$ ) over the ND surface $S$ in contact with the substrate leads to $V \cdot C_{A u} \cdot d \Delta T_{p} / d t=G \cdot S \cdot \Delta T_{p}$. This results in an exponential decay of $\Delta T_{p}$ with a characteristic time $\tau=c_{A u} h / G, C_{A u}$ being the volumetric heat capacity of gold and $G$ the Kapitza thermal conductance of the nanostructure/substrate interface. ${ }^{51}$ Such an exponential decay, with a characteristic time linearly scaling with ND height, has been experimentally reported for permalloy NDs nanopatterned on a Si substrate ${ }^{10}$ and the transient thermal dynamics theoretically investigated for the case of $\mathrm{Cu}$ NDs deposited on $\mathrm{Si}^{52}$. Within the context of the present study, the $\tau_{1} \propto \mathrm{h}$ scaling is in approximate agreement with the $2.4 \mathrm{x}$ smaller $\tau_{1}$ values determined for $\mathrm{h}=18 \mathrm{~nm}$ NDs as compared to $\mathrm{h}=40 \mathrm{~nm}$ ones, and their weak dependence on ND diameter. Furthermore, preliminary $\mathrm{G}$ estimation via $\mathrm{G}=\mathrm{c}_{\mathrm{Au}} \mathrm{h} / \tau$ leads to $\mathrm{G}$ values in the order of $60 \mathrm{MW} \cdot \mathrm{m}^{-2} \cdot \mathrm{K}^{-1}$ for the gold-sapphire interface, which is consistent with the $20-70 \mathrm{MW} \cdot \mathrm{m}^{-2} \cdot \mathrm{K}^{-1}$ range reported in the literature for thin gold films. ${ }^{6,53-56}$

A more thorough modeling ${ }^{52}$ of ND cooling dynamics requires to include the effect of the finite heat conductivity $\Lambda_{m}$ of the sapphire substrate (associated to a $J_{m}=-\Lambda_{m} \operatorname{grad}\left(T_{m}\right)$ heat current density according to Fourier law, Fig. 4b), which was achieved using FEM numerical simulations (described in the Methods part). The dynamics computed using the complete FEM model for NDs with 18 or $40 \mathrm{~nm}$ thicknesses and 80 or $160 \mathrm{~nm}$ diameters (solid lines in Fig. 4c) are slightly slower than the purely 
exponential ones with $\tau_{1}=\mathrm{C}_{\text {Au }} \mathrm{h} / \mathrm{G}$ decay time obtained by assuming infinitely fast heat diffusion in the substrate for the same $\mathrm{G}=70 \mathrm{MW} \cdot \mathrm{m}^{-2} \cdot \mathrm{K}^{-1}$ considered value of interface conductance (dashed lines), the difference increasing with ND diameter. They also deviate from a purely exponential decay, as also observed in a previous theoretical work addressing the cooling of a metal ND in contact with a dielectric substrate, in the case where the latter does not remain isothermal during the cooling process. ${ }^{52}$ However, their non-exponential character is noticeable only at time scales exceeding those considered in the experiments. The fact that ND cooling dynamics are primarily limited by heat transfer at the ND-substrate interface is confirmed by comparing the cooling dynamics computed by assuming a purely interface-limited process (corresponding to infinite $\Lambda_{\mathrm{A} 1203}$ sapphire thermal conductivity, dashed lines in Fig. 4c) and a purely diffusion-limited process (corresponding to infinite G, dotted lines in Fig. 4c). As expected, the latter dynamics are strongly non-exponential, dependent on ND diameter and considerably faster than the former ones for the whole range of ND sizes explored in the experiments. It is however interesting to note that the large difference between the time scales associated to interfacial heat transfer and heat diffusion in the sapphire substrate demonstrated here for gold NDs would be strongly reduced for some other NDs compositions allowing better interface conductance with the substrate, or for nanoparticles with larger lateral sizes (or nanostripes or thin films). Regarding ND composition, interfacial heat transfer would be for instance about 5 times faster for a nickel ND than for a gold one, as $\mathrm{G} \approx 300 \mathrm{MW} \cdot \mathrm{m}^{-2} \cdot \mathrm{K}^{-1}$ for a nickel-sapphire interface ${ }^{56}$ Regarding nanoparticle size, Fig. 4c shows that increasing ND diameter increases the thermal cooling time scales. The less efficient cooling predicted for a heat source with large lateral size (the limiting case corresponding to a continuous film, i.e. $\mathrm{D} / \mathrm{h}>>1$ ) as compared to the smallest ones, can be understood from the fact that in the latter case of a small nano-object heat can spread inside the substrate in three dimensions (hot spot behavior), while in the former case it mainly propagates in the direction perpendicular to the interface (the less efficient dissipation of the heat generated close to the interface in the former case leading to higher temperature rises in this region).

The difference between the dynamics computed using the complete FEM model and in the simple interface-limited case (in which substrate temperature remains uniform and unmodified throughout the cooling process) implies that extracting a precise $G$ value from time-resolved experimental data requires their comparison with results of the complete FEM model, especially for the NDs with the largest diameter values, i.e. approaching the continuum film case (for which the isothermal substrate hypothesis is less satisfied). To do so, the measured cooling times $\tau_{1}$ were compared to those extracted from exponential fits of the FEM-simulated cooling dynamics using different $G$ values (Fig. $4 \mathrm{~d}$ ). The cooling times determined for $\mathrm{h}=40 \mathrm{~nm}$ NDs are well reproduced by calculations assuming $\mathrm{G}=(60 \pm 10) \mathrm{MW} \cdot \mathrm{m}^{-2} \cdot \mathrm{K}^{-1}$. Those of $\mathrm{h}=18 \mathrm{~nm}$ NDs correspond to a slightly larger 
$\mathrm{G}=(75 \pm 10) \mathrm{MW} \cdot \mathrm{m}^{-2} \cdot \mathrm{K}^{-1}$ average value. The small difference between the $\mathrm{G}$ values extracted from the two ND thicknesses, corresponding to two distinct samples fabricated during two different processing sessions, could be due to either small differences in the gold-sapphire interface in these two samples or to uncertainties associated to the determination of their average thicknesses by AFM.

\section{CONCLUSIONS}

In conclusion, the cooling dynamics of individual supported nanoparticles (gold NDs with 60$190 \mathrm{~nm}$ diameters and 18-40 nm thicknesses) were quantitatively investigated using an optical pumpprobe spectroscopy approach combined with a spatial modulation microscope. The use of moderate pump fluences, limiting lattice heating to $<15 \mathrm{~K}$ values, was essential to avoid permanent modification of the measured signals during the experiments. The absolute amplitudes of the transient extinction cross-section changes, induced by ND heating, were seen to strongly depend on probe wavelength, in good agreement with the results of a thermo-optical model. All experimental signals measured on NDs with diameters down to $60 \mathrm{~nm}$ could be theoretically reproduced using Fourier law with bulk sapphire thermal properties, and a gold-sapphire thermal interface conductance of about $70 \mathrm{MW} \cdot \mathrm{m}^{-2} \cdot \mathrm{K}^{-1}$ showing weak size dependence in the investigated size range and consistent with previous measurements on thin films. As demonstrated by FEM investigations, the cooling dynamics measured here are mostly limited by the gold-sapphire interface, the characteristic times associated with interfacial heat transfer from the ND to the substrate being considerably longer than those associated with substrate heat diffusion. These experiments may be extended to other compositions and geometries of the nano-objects and their supporting substrate, opening the way to direct investigations of the different nanoscale thermal transport regimes at the single-particle level.

\section{AUTHOR INFORMATION}

\section{Corresponding author}

*E-mail: aurelien.crut@univ-lyon1.fr

\section{ORCID}

Fabio Medeghini : 0000-0002-0508-3365

Paolo Maioli: 0000-0002-4199-8810

Francesco Rossella: 0000-0002-0601-4927

Francesco Banfi: 0000-0002-7465-8417

Aurélien Crut: 0000-0003-2185-709X

Natalia Del Fatti: 0000-0002-8074-256X

\section{Present Addresses}


† F. M.: JILA, University of Colorado Boulder and National Institute of Standard and Technology, Boulder, Colorado 80309, USA

\section{Notes}

The authors declare no competing financial interest.

\section{ACKNOWLEDGMENTS}

This work was supported by the LABEX iMUST (ANR-10-LABX-0064) of Université de Lyon, within the program "Investissements d'Avenir" (ANR-11-IDEX-0007) operated by the French National Research Agency (ANR). The authors thank Antoine Gallet and Marco Gandolfi for their help at initial stages of the project and Fabien Vialla for useful discussions. Francesco Banfi acknowledges financial support from Université de Lyon in the frame of the IDEXLYON Project -Programme Investissements d' Avenir (ANR-16-IDEX-0005) and from Université Claude Bernard Lyon 1 through the BQR Accueil EC 2019 grant.

\section{REFERENCES}

(1) Cahill, D. G.; Ford, W. K.; Goodson, K. E.; Mahan, G. D.; Majumdar, A.; Maris, H. J.; Merlin, R.; Phillpot, S. R. Nanoscale Thermal Transport. J. Appl. Phys. 2003, 93, 793-818.

(2) Cahill, D. G.; Braun, P. V.; Chen, G.; Clarke, D. R.; Fan, S.; Goodson, K. E.; Keblinski, P.; King, W. P.; Mahan, G. D.; Majumdar, A.; et al. Nanoscale Thermal Transport. II. 2003-2012. Appl. Phys. Rev. 2014, 1, 011305.

(3) Pop, E.; Sinha, S.; Goodson, K. E. Heat Generation and Transport in Nanometer-Scale Transistors. Proc. IEEE 2006, 94, 1587-1601.

(4) Snyder, G. J.; Toberer, E. S. Complex Thermoelectric Materials. Nat. Mater. 2008, 7, 105-114.

(5) Swartz, E.; Pohl, R. Thermal Boundary Resistance. Rev. Mod. Phys. 1989, 61, 605-668.

(6) Stoner, R. J.; Maris, H. J. Kapitza Conductance and Heat Flow between Solids at Temperatures from 50 to 300 K. Phys. Rev. B 1993, 48, 16373-16387.

(7) Wilson, O. M.; Hu, X.; Cahill, D. G.; Braun, P. V. Colloidal Metal Particles as Probes of Nanoscale Thermal Transport in Fluids. Phys. Rev. B 2002, 66, 224301.

(8) Juvé, V.; Scardamaglia, M.; Maioli, P.; Crut, A.; Merabia, S.; Joly, L.; Del Fatti, N.; Vallée, F. Cooling Dynamics and Thermal Interface Resistance of Glass-Embedded Metal Nanoparticles. 
Phys. Rev. B 2009, 80, 195406.

(9) Giannetti, C.; Revaz, B.; Banfi, F.; Montagnese, M.; Ferrini, G.; Cilento, F.; Maccalli, S.; Vavassori, P.; Oliviero, G.; Bontempi, E.; et al. Thermomechanical Behavior of Surface Acoustic Waves in Ordered Arrays of Nanodisks Studied by Near-Infrared Pump-Probe Diffraction Experiments. Phys. Rev. B 2007, 76, 125413.

(10) Giannetti, C.; Banfi, F.; Nardi, D.; Ferrini, G.; Parmigiani, F. Ultrafast Laser Pulses to Detect and Generate Fast Thermomechanical Transients in Matter. IEEE Photonics J. 2009, 1, 21-32.

(11) Chen, G. Nonlocal and Nonequilibrium Heat Conduction in the Vicinity of Nanoparticles. J. Heat Transfer 1996, 118, 539-545.

(12) Siemens, M. E.; Li, Q.; Yang, R.; Nelson, K. A.; Anderson, E. H.; Murnane, M. M.; Kapteyn, H. C. Quasi-Ballistic Thermal Transport from Nanoscale Interfaces Observed Using Ultrafast Coherent Soft X-Ray Beams. Nat. Mater. 2010, 9, 26-30.

(13) Gandolfi, M.; Benetti, G.; Glorieux, C.; Giannetti, C.; Banfi, F. International Journal of Heat and Mass Transfer Accessing Temperature Waves : A Dispersion Relation Perspective. Int. J. Heat Mass Transf. 2019, 143, 118553.

(14) Simoncelli, M.; Marzari, N.; Cepellotti, A. Generalization of Fourier's Law into Viscous Heat Equations. Phys. Rev. X 2020, 10, 11019.

(15) Schmidt, A. J.; Cheaito, R.; Chiesa, M. A Frequency-Domain Thermoreflectance Method for the Characterization of Thermal Properties. Rev. Sci. Instrum. 2009, 80.

(16) Regner, K. T.; Sellan, D. P.; Su, Z.; Amon, C. H.; McGaughey, A. J. H.; Malen, J. A. Broadband Phonon Mean Free Path Contributions to Thermal Conductivity Measured Using Frequency Domain Thermoreflectance. Nat. Commun. 2013, 4, 1640.

(17) Wilson, R. B.; Cahill, D. G. Anisotropic Failure of Fourier Theory in Time-Domain Thermoreflectance Experiments. Nat. Commun. 2014, 5, 1-11.

(18) Minnich, A. J.; Johnson, J. A.; Schmidt, A. J.; Esfarjani, K.; Dresselhaus, M. S.; Nelson, K. A.; Chen, G. Thermal Conductivity Spectroscopy Technique to Measure Phonon Mean Free Paths. Phys. Rev. Lett. 2011, 107, 095901.

(19) Johnson, J. A.; Maznev, A. A.; Cuffe, J.; Eliason, J. K.; Minnich, A. J.; Kehoe, T.; Torres, C. M. S.; Chen, G.; Nelson, K. A. Direct Measurement of Room-Temperature Nondiffusive Thermal Transport Over Micron Distances in a Silicon Membrane. Phys. Rev. Lett. 2013, 110, 025901. 
(20) Hu, M.; Hartland, G. V. Heat Dissipation for Au Particles in Aqueous Solution: Relaxation Time versus Size. J. Phys. Chem. B 2002, 106, 7029-7033.

(21) Stoll, T.; Maioli, P.; Crut, A.; Rodal-Cedeira, S.; Pastoriza-Santos, I.; Vallée, F.; Del Fatti, N. Time-Resolved Investigations of the Cooling Dynamics of Metal Nanoparticles: Impact of Environment. J. Phys. Chem. C 2015, 119, 12757-12764.

(22) Park, J.; Cahill, D. G. Plasmonic Sensing of Heat Transport at Solid-Liquid Interfaces. J. Phys. Chem. C 2016, 120, 2814-2821.

(23) Banfi, F.; Juvé, V.; Nardi, D.; Dal Conte, S.; Giannetti, C.; Ferrini, G.; Del Fatti, N.; Vallée, F. Temperature Dependence of the Thermal Boundary Resistivity of Glass-Embedded Metal Nanoparticles. Appl. Phys. Lett. 2012, 100, 011902.

(24) Gandolfi, M.; Crut, A.; Medeghini, F.; Stoll, T.; Maioli, P.; Vallée, F.; Banfi, F.; Del Fatti, N. Ultrafast Thermo-Optical Dynamics of Plasmonic Nanoparticles. J. Phys. Chem. C 2018, 122, 8655-8666.

(25) Ferrera, M.; Della Valle, G.; Sygletou, M.; Magnozzi, M.; Catone, D.; O’Keeffe, P.; Paladini, A.; Toschi, F.; Mattera, L.; Canepa, M.; et al. Thermometric Calibration of the Ultrafast Relaxation Dynamics in Plasmonic Au Nanoparticles. ACS Photonics 2020, 7, 959-966.

(26) Hoogeboom-Pot, K. M.; Hernandez-Charpak, J. N.; Gu, X.; Frazer, T. D.; Anderson, E. H.; Chao, W.; Falcone, R. W.; Yang, R.; Murnane, M. M.; Kapteyn, H. C.; et al. A New Regime of Nanoscale Thermal Transport: Collective Diffusion Increases Dissipation Efficiency. Proc. Natl. Acad. Sci. 2015, 112, 4846-4851.

(27) Frazer, T. D.; Knobloch, J. L.; Hoogeboom-Pot, K. M.; Nardi, D.; Chao, W.; Falcone, R. W.; Murnane, M. M.; Kapteyn, H. C.; Hernandez-Charpak, J. N. Engineering Nanoscale Thermal Transport: Size- and Spacing-Dependent Cooling of Nanostructures. Phys. Rev. Appl. 2019, 11, 024042.

(28) Hu, Y.; Zeng, L.; Minnich, A. J.; Dresselhaus, M. S.; Chen, G. Spectral Mapping of Thermal Conductivity through Nanoscale Ballistic Transport. Nat. Nanotechnol. 2015, 10, 701-706.

(29) Zhang, H.; Hua, C.; Ding, D.; Minnich, A. J. Length Dependent Thermal Conductivity Measurements Yield Phonon Mean Free Path Spectra in Nanostructures. Sci. Rep. 2015, 5, 9121.

(30) Crut, A.; Maioli, P.; Del Fatti, N.; Vallée, F. Optical Absorption and Scattering Spectroscopies of Single Nano-Objects. Chem. Soc. Rev. 2014, 43, 3921. 
(31) Hu, M.; Novo, C.; Funston, A.; Wang, H.; Staleva, H.; Zou, S.; Mulvaney, P.; Xia, Y.; Hartland, G. V. Dark-Field Microscopy Studies of Single Metal Nanoparticles: Understanding the Factors That Influence the Linewidth of the Localized Surface Plasmon Resonance. J. Mater. Chem. 2008, 18, 1949-1960.

(32) Baida, H.; Mongin, D.; Christofilos, D.; Bachelier, G.; Crut, A.; Maioli, P.; Del Fatti, N.; Vallée, F. Ultrafast Nonlinear Optical Response of a Single Gold Nanorod near Its Surface Plasmon Resonance. Phys. Rev. Lett. 2011, 107, 057402.

(33) Ruijgrok, P. V; Zijlstra, P.; Tchebotareva, A. L.; Orrit, M. Damping of Acoustic Vibrations of Single Gold Nanoparticles Optically Trapped in Water. Nano Lett. 2012, 12, 1063-1069.

(34) Staleva, H.; Hartland, G. V. Vibrational Dynamics of Silver Nanocubes and Nanowires Studied by Single-Particle Transient Absorption Spectroscopy. Adv. Funct. Mater. 2008, 18, 38093817.

(35) Crut, A.; Maioli, P.; Del Fatti, N.; Vallée, F. Acoustic Vibrations of Metal Nano-Objects: TimeDomain Investigations. Phys. Rep. 2015, 549, 1-43.

(36) Medeghini, F.; Crut, A.; Gandolfi, M.; Rossella, F.; Maioli, P.; Vallée, F.; Banfi, F.; Del Fatti, N. Controlling the Quality Factor of a Single Acoustic Nanoresonator by Tuning Its Morphology. Nano Lett. 2018, 18, 5159-5166.

(37) Medeghini, F.; Rouxel, R.; Crut, A.; Maioli, P.; Rossella, F.; Banfi, F.; Vallée, F.; Del Fatti, N. Signatures of Small Morphological Anisotropies in the Plasmonic and Vibrational Responses of Individual Nano-Objects. J. Phys. Chem. Lett. 2019, 10, 5372-5380.

(38) Arbouet, A.; Christofilos, D.; Del Fatti, N.; Vallée, F.; Huntzinger, J.; Arnaud, L.; Billaud, P.; Broyer, M. Direct Measurement of the Single-Metal-Cluster Optical Absorption. Phys. Rev. Lett. 2004, 93, 127401.

(39) Muskens, O. L.; Del Fatti, N.; Vallée, F. Femtosecond Response of a Single Metal Nanoparticle. Nano Lett. 2006, 6, 552-556.

(40) Johnson, P. B.; Christy, R. W. Optical Constants of the Noble Metals. Phys. Rev. B 1972, 6, 4370-4379.

(41) Aspnes, D. E.; Kinsbron, E.; Bacon, D. D. Optical Properties of Au: Sample Effects. Phys. Rev. B 1980, 21, 3290-3299.

(42) Olmon, R. L.; Slovick, B.; Johnson, T. W.; Shelton, D.; Oh, S.-H.; Boreman, G. D.; Raschke, M. B. 
Optical Dielectric Function of Gold. Phys. Rev. B 2012, 86, 235147.

(43) Thomas, M. E.; Andersson, S. K.; Sova, R. M.; Joseph, R. I. Frequency and Temperature Dependence of the Refractive Index of Sapphire. Infrared Phys. Technol. 1998, 39, 235-249.

(44) Wilson, R. B.; Apgar, B. A.; Martin, L. W.; Cahill, D. G. Thermoreflectance of Metal Transducers for Optical Pump-Probe Studies of Thermal Properties. Opt. Express 2012, 20, 28829-28838.

(45) Stoll, T.; Maioli, P.; Crut, A.; Del Fatti, N.; Vallée, F. Advances in Femto-Nano-Optics: Ultrafast Nonlinearity of Metal Nanoparticles. Eur. Phys. J. B 2014, 87, 260.

(46) Chen, Y. J.; Lee, M. C.; Wang, C. M. Dielectric Function Dependence on Temperature for Au and Ag. Jpn. J. Appl. Phys. 2014, 53.

(47) Shen, P.-T.; Sivan, Y.; Lin, C.-W.; Liu, H.-L.; Chang, C.-W.; Chu, S.-W. Temperature- and Roughness-Dependent Permittivity of Annealed/Unannealed Gold Films. Opt. Express 2016, 24, 19254.

(48) Reddy, H.; Guler, U.; Kildishev, A. V.; Boltasseva, A.; Shalaev, V. M. Temperature-Dependent Optical Properties of Gold Thin Films. Opt. Mater. Express 2016, 6, 2776.

(49) Zhang, S.; Pei, Y.; Liu, L. Dielectric Function of Polycrystalline Gold Films: Effects of Grain Boundary and Temperature. J. Appl. Phys. 2018, 124.

(50) Magnozzi, M.; Ferrera, M.; Mattera, L.; Canepa, M.; Bisio, F. Plasmonics of Au Nanoparticles in a Hot Thermodynamic Bath. Nanoscale 2019, 11, 1140-1146.

(51) Caddeo, C.; Melis, C.; Ronchi, A.; Giannetti, C.; Ferrini, G.; Rurali, R.; Colombo, L.; Banfi, F. Thermal Boundary Resistance from Transient Nanocalorimetry: A Multiscale Modeling Approach. Phys. Rev. B 2017, 95, 085306.

(52) Banfi, F.; Pressacco, F.; Revaz, B.; Giannetti, C.; Nardi, D.; Ferrini, G.; Parmigiani, F. Ab Initio Thermodynamics Calculation of All-Optical Time-Resolved Calorimetry of Nanosize Systems: Evidence of Nanosecond Decoupling of Electron and Phonon Temperatures. Phys. Rev. B 2010, 81, 155426.

(53) Xu, Y.; Wang, H.; Tanaka, Y.; Shimono, M.; Yamazaki, M. Measurement of Interfacial Thermal Resistance by Periodic Heating and a Thermo-Reflectance Technique. Mater. Trans. 2007, 48, 148-150.

(54) Freedman, J. P.; Yu, X.; Davis, R. F.; Gellman, A. J.; Malen, J. A. Thermal Interface Conductance across Metal Alloy-Dielectric Interfaces. Phys. Rev. B 2016, 93, 035309. 
(55) Blank, M.; Weber, L. Influence of the Thickness of a Nanometric Copper Interlayer on Au/Dielectric Thermal Boundary Conductance. J. Appl. Phys. 2018, 124, 105304.

(56) Blank, M.; Weber, L. Towards a Coherent Database of Thermal Boundary Conductance at Metal/Dielectric Interfaces. J. Appl. Phys. 2019, 125, 095302. 


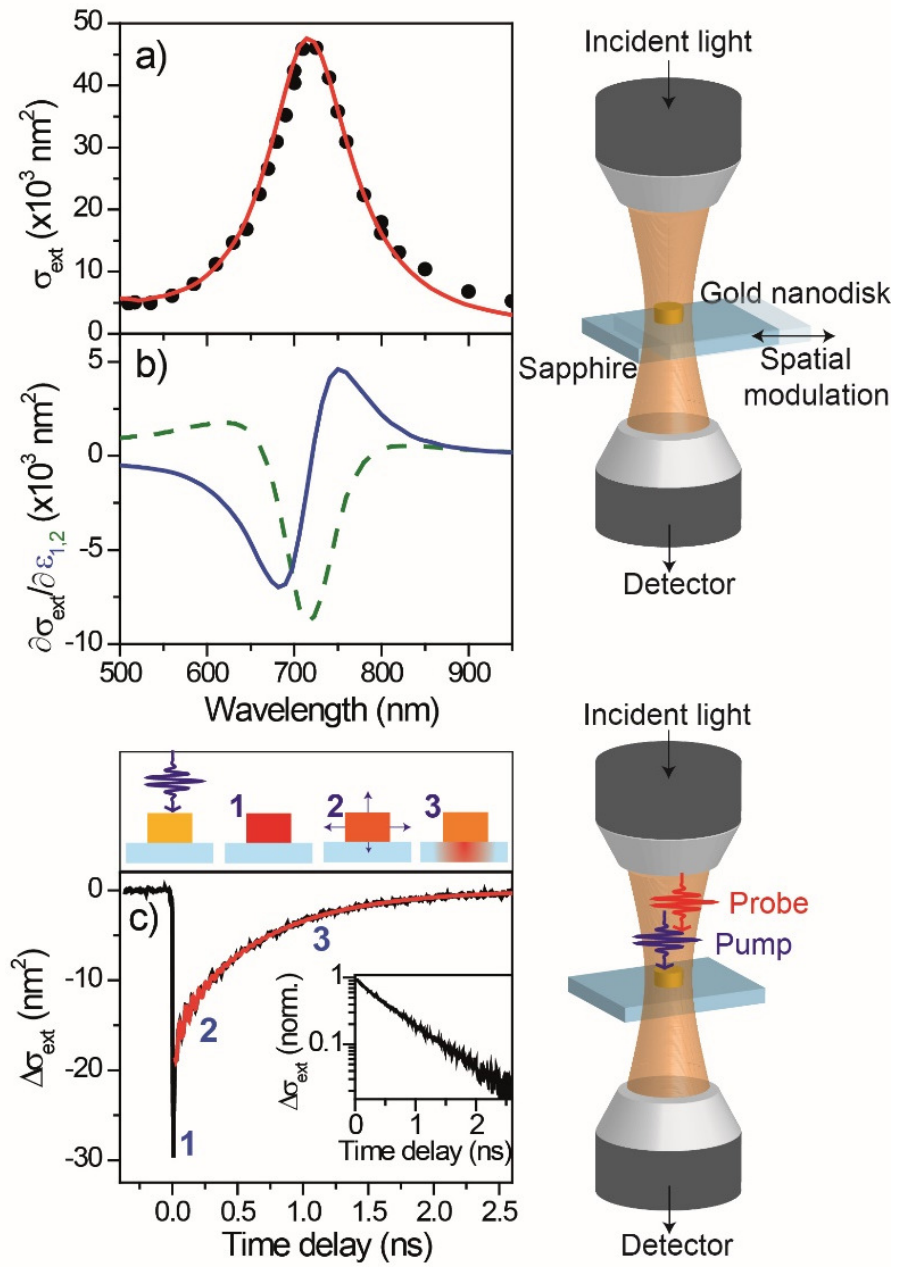

Figure 1. Linear and time-resolved optical spectroscopies of an individual gold ND supported on a sapphire substrate. a) Absolute extinction spectrum of a single ND (referred to as ND1, black dots) measured by spatial modulation spectroscopy, whose principle is shown on the right (see also the main text). ND1 extinction spectrum is well reproduced by FEM calculations performed with $D=101 \mathrm{~nm}$ and $\mathrm{h}=21 \mathrm{~nm}$ ND diameter and thickness (red line). b) $\partial \sigma_{\mathrm{ext}} / \partial \varepsilon_{1}$ (blue) and $\partial \sigma_{\mathrm{ext}} / \partial \varepsilon_{2}$ (green) derivatives deduced from FEM calculations. c) Ultrafast extinction changes of ND1 following its impulsive excitation, measured using time-resolved pump-probe spectroscopy (principle shown on the right) with $\lambda_{p p}=410 \mathrm{~nm}$ pump wavelength, $\lambda_{\mathrm{pr}}=690 \mathrm{~nm}$ probe wavelength and $0.7 \mathrm{~J} . \mathrm{m}^{-2}$ pump fluence. The signal contains signatures of three physical processes: ND excitation and internal thermalization (1), damped acoustic vibrations (2) and cooling (3). The normalized thermal part of the signal, obtained by subtraction of the contributions of the two former processes, is shown in the inset on a semilogarithmic scale, highlighting its quasi-exponential character. 

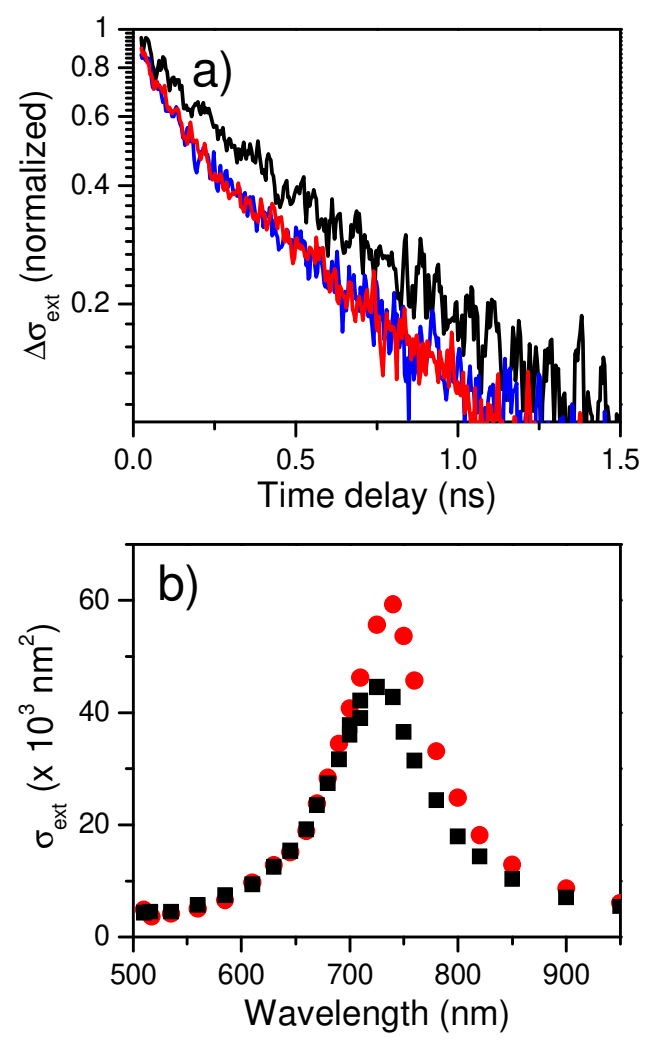

Figure 2. Pump fluence effect. a) Thermal component of the transient optical responses successively measured on ND2 (a ND made with the same nanofabrication parameters as ND1) using $\lambda_{p p}=410 \mathrm{~nm}$, $\lambda_{\mathrm{pr}}=515 \mathrm{~nm}$ and pump fluences of $\mathrm{F}_{\mathrm{pp}}=1 \mathrm{~J} \cdot \mathrm{m}^{-2}$ (black line), $2.7 \mathrm{~J} \cdot \mathrm{m}^{-2}$ (red line) and $1 \mathrm{~J} \cdot \mathrm{m}^{-2}$ again (blue line), demonstrating the permanent modification of ND2 ultrafast response after exposure to high pump fluences. b) SMS-measured extinction spectra of ND2 before (black squares) and after the pumpprobe experiments with high pump fluence (red circles). 

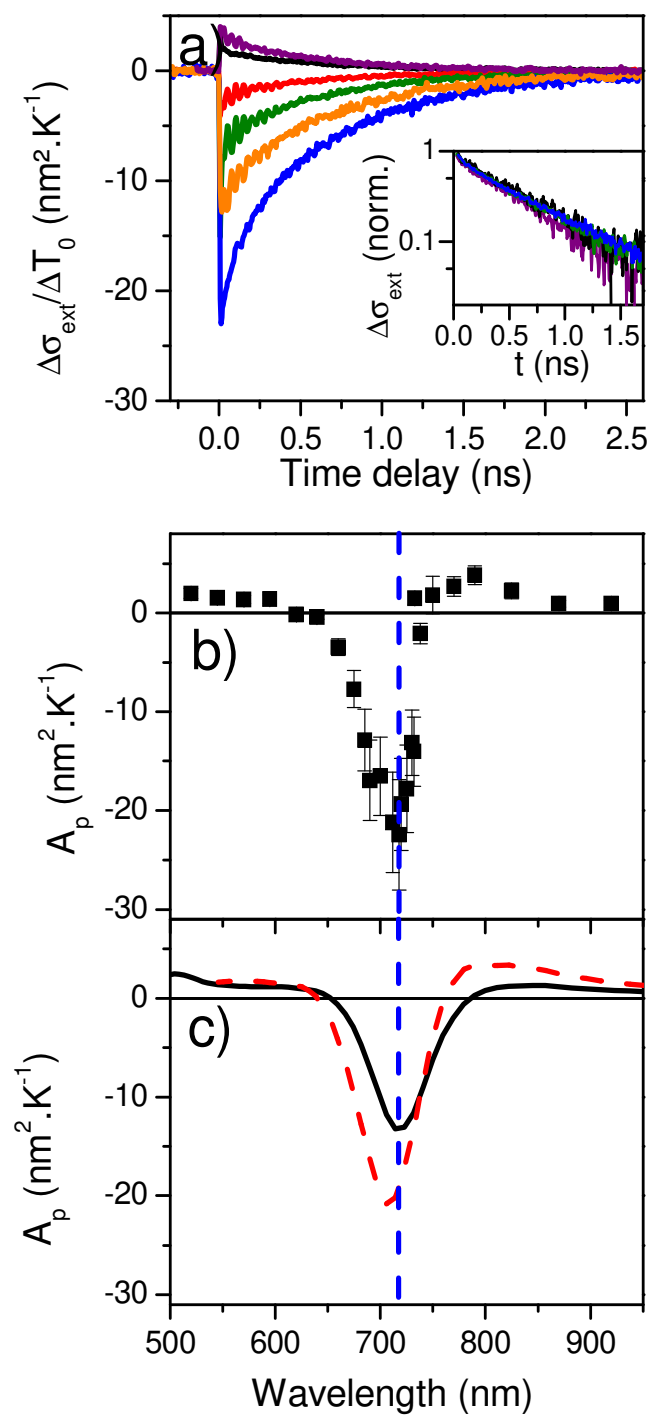

Figure 3. Probe wavelength effect on the measured time-resolved signals. a) Time-resolved signals measured on ND1 with probe wavelengths $\lambda_{\mathrm{pr}}=520 \mathrm{~nm}$ (black), $660 \mathrm{~nm}$ (red), $675 \mathrm{~nm}$ (green), $718 \mathrm{~nm}$ (blue), $730 \mathrm{~nm}$ (orange) (with $\lambda_{\mathrm{pp}}=410 \mathrm{~nm}$ for all these signals) and $790 \mathrm{~nm}$ (purple, with $\lambda_{\mathrm{pp}}=395$ $\mathrm{nm}$ ), divided by the estimated initial ND temperature rise $\Delta \mathrm{T}_{0}$. The normalized thermal components of these signals are shown in the inset in lin-log scale (same color code). b) $A_{p}$ coefficients deduced from the amplitude of the thermal components of time-resolved signals measured on ND1 (eq. 1). c) $A_{p}$ coefficients computed based on eq. 2 using the $\partial \sigma_{\text {ext }} / \partial \varepsilon_{1}$ and $\partial \sigma_{\text {ext }} / \partial \varepsilon_{2}$ spectra deduced from FEM calculations (Fig. 1b) and d $\varepsilon_{1,2} / \mathrm{dT}$ values taken from refs. ${ }^{44}$ (solid black line) and ${ }^{45}$ (dashed red line). The dashed blue line indicates the central position of the SPR. 

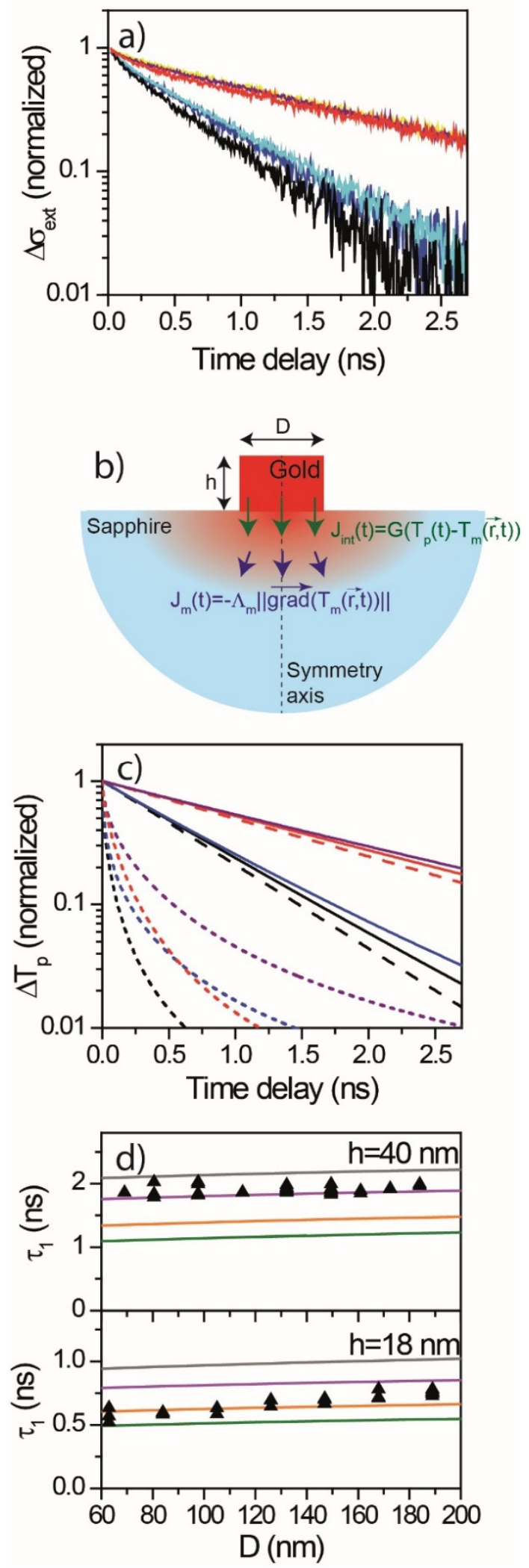

Figure 4. Morphological dependence of the cooling dynamics of individual gold NDs. a) Normalized thermal components of experimental time-resolved signals measured on individual gold NDs with $\mathrm{h}=18$ $\mathrm{nm}$ thickness (estimated by AFM) and diameters of about 60 (black), 120 (blue) and $180 \mathrm{~nm}$ (cyan), and $\mathrm{h}=40 \mathrm{~nm}$ thickness and diameters of about 70 (red), 120 (purple) and $180 \mathrm{~nm}$ (yellow) (estimated 
by SEM). b) Schematics of the FEM simulations, showing the geometry and the two cooling processes (heat transfer at the interface and in the substrate, with associated heat current densities $\mathrm{J}_{\text {int }}$ and $\mathrm{J}_{\mathrm{m}}$ ) considered. c) FEM-computed dynamics for $h=18 \mathrm{~nm}, D=80 \mathrm{~nm}$ (black), $h=18 \mathrm{~nm}, D=160 \mathrm{~nm}$ (blue), $\mathrm{h}=40 \mathrm{~nm}, \mathrm{D}=80 \mathrm{~nm}$ (red) and $\mathrm{h}=40 \mathrm{~nm}, \mathrm{D}=160 \mathrm{~nm}$ (purple) NDs (plain lines), assuming a $\mathrm{G}=70 \mathrm{MW} \cdot \mathrm{m}^{-}$ ${ }^{2} . \mathrm{K}^{-1}$ conductance at the gold-sapphire interface. The cooling dynamics computed for these NDs in the $\Lambda_{\mathrm{Al} 203} \rightarrow \infty$ and $\mathrm{G} \rightarrow \infty$ limits are also shown as dashed and dotted lines, respectively. Note that only two lines are shown in the former case, for which the cooling dynamics only depend on ND thickness. d) Summary of the $\tau_{1}$ cooling times measured on $\mathrm{h}=40 \mathrm{~nm}$ and $\mathrm{h}=18 \mathrm{~nm}$ gold NDs (black triangles, each one corresponding to a different gold ND). FEM-computed cooling times for $\mathrm{G}=50$ (grey), 60 (magenta), 80 (orange) and $100 \mathrm{MW} \cdot \mathrm{m}^{-2} \cdot \mathrm{K}^{-1}$ (green) are also shown. 
TOC

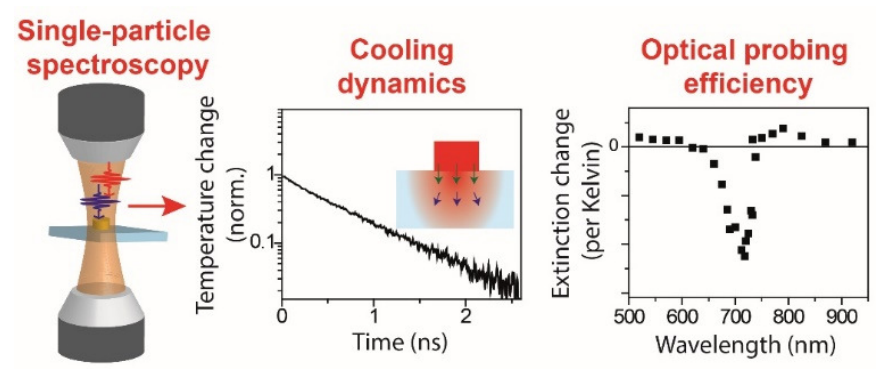

